\title{
Remote video-based outcome measures of patients with Parkinson's disease after deep brain stimulation using smartphones: a pilot study
}

\author{
Xinmeng Xu, BS, ${ }^{1}$ Zhitong Zeng, MD, ${ }^{2}$ Yijia Qi, BS, ${ }^{1}$ Kang Ren, PhD, ${ }^{3}$ \\ Chencheng Zhang, MD, PhD, ${ }^{2,4}$ Bomin Sun, MD, PhD, ${ }^{2}$ and Dianyou Li, MD, PhD ${ }^{2}$
}

\begin{abstract}
${ }^{1}$ Clinical Neuroscience Center, Ruijin Hospital Luwan Branch, Shanghai Jiao Tong University School of Medicine, Shanghai; ${ }^{2}$ Department of Neurosurgery, Center for Functional Neurosurgery, Ruijin Hospital, Shanghai Jiao Tong University School of Medicine, Shanghai; ${ }^{3}$ GYENNO SCIENCE CO., LTD., Shenzhen; and ${ }^{4}$ Shanghai Research Center for Brain Science and BrainInspired Intelligence, Shanghai, China
\end{abstract}

OBJECTIVE To provide better postoperative healthcare for patients with Parkinson's disease (PD) who received deep brain stimulation (DBS) surgery and to allow surgeons improved tracking of surgical outcomes, the authors sought to examine the applicability and feasibility of remote assessment using smartphones.

METHODS A disease management mobile application specifically for PD was used to perform the remote assessment of patients with PD who underwent DBS. Connection with patients was first established via a phone call or a social application, and instructions for completing the remote assessment were delivered. During the video-based virtual meeting, three nonmotor assessment scales measuring the quality of life and mental state, and a modified version of the Movement Disorder Society-sponsored revision of the Unified Parkinson's Disease Rating Scale, part III (MDS-UPDRS III) measuring motor abilities were evaluated. After the assessment, a report and the satisfaction questionnaire were sent to the patient.

RESULTS Overall, 22 patients were recruited over a 4-week period. Among those, 18 patients completed the assessment on the mobile application. The mean duration was 41.3 minutes for video assessment and 17.5 minutes for nonmotor assessment via telephone. The mean estimated cost was 427.68 Chinese yuan (CNY) for an in-person visit and $20.91 \mathrm{CNY}$ for a virtual visit $(p<0.001)$. The mean time estimate for an in-person visit was 5.51 hours and 0.68 hours for a virtual visit $(p=0.002)$. All patients reported satisfaction $(77.78 \%$ very satisfied and $22.22 \%$ satisfied) with the virtual visit and were specifically impressed by the professionalism and great attitude of the physician assistant. The majority of patients agreed that the evaluation time was reasonable (50\% totally agree, $44.44 \%$ agree, and $5.56 \%$ neither agree nor disagree) and all patients expressed interest in future virtual visits ( $61.11 \%$ very willingly and $38.89 \%$ willingly). No adverse events were observed during the virtual visit.

CONCLUSIONS Innovation in remote assessment technologies was highly feasible for its transforming power in the clinical management of patients with PD who underwent DBS and research. Video-based remote assessment offered considerable time and resource reduction for both patients and doctors. It also increased safety and was a well-accepted, favored tool. Finally, the results of this study have shown there is potential to combine remote assessment tools with real-life clinical visits and other telemedical technologies to collectively benefit the postoperative healthcare of patients with PD undergoing DBS.

https://thejns.org/doi/abs/10.3171/2021.8.FOCUS21383

KEYWORDS Parkinson's disease; deep brain stimulation; telemedicine; remote assessment; virtual videoconferencing; smartphone

$\mathrm{P}$ ARKINSON's disease (PD) is the second most prevalent neurodegenerative disease, with more than 6.1 million people affected by $2016 .^{1,2}$ Among the motor, cognitive, and emotional disturbances induced by $\mathrm{PD}$, motor symptoms cause the most substantial negative impact, which can sometimes be life-threatening. ${ }^{3}$ In addition to medication, deep brain stimulation (DBS) is a wellestablished treatment for patients with PD and has been chosen by more than 100,000 patients. ${ }^{4,5}$

Such a high prevalence of PD, coupled with the severe

ABBREVIATIONS CNY = Chinese yuan; DBS = deep brain stimulation; H-Y = Hoehn and Yahr; MDS-UPDRS III = Movement Disorder Society-sponsored revision of the Unified Parkinson's Disease Rating Scale, part III; MMSE = Mini-Mental State Examination; PD = Parkinson's disease; PDQ-8 = Parkinson's Disease Questionnaire 8. ACCOMPANYING EDITORIAL DOI: 10.3171/2021.8.FOCUS21492

SUBMITTED June 30, 2021. ACCEPTED August 17, 2021.

INCLUDE WHEN CITING DOI: 10.3171/2021.8.FOCUS21383. 
outcomes of motor deficiency, yields strong demands in the management of PD. Optimal PD DBS management requires contributions from a multidisciplinary team, including patients, surgeons, neurologists, physician assistants, psychiatrists, and rehabilitation specialists. ${ }^{1}$ Maintaining an optimal treatment effect can require a demanding amount of commitment from patients, caregivers, and doctors. ${ }^{6-10}$ Further demands placed on the patient include high travel expenses, lack of companionship, and physical immobility. ${ }^{11}$ Furthermore, the onset of the COVID-19 pandemic has largely halted in-person visits, especially for the elderly (aged $\geq 65$ years) because of the higher infection risk. ${ }^{1,12}$ It has disrupted patients' regular follow-ups with the doctor and has hindered the surgeon from tracking surgical outcomes. Thus, telemedicine, which consists of teleconsultation, telemonitoring, and teletreatment, has been rapidly adopted as a supportive development for healthcare professionals to provide remote care. ${ }^{1,13}$ Researchers have investigated the feasibility, reliability, and cost-effectiveness of remote assessment and have expanded on various aspects of telemedicine such as teleprogramming and the application of wearable sensors. ${ }^{14-18}$ However, teleconsultation for patients with PD who have opted for DBS treatment is still lacking ${ }^{19}$ (systematic literature search results on PubMed are listed in Supplemental Table 1).

Multidisciplinary teams have collectively contributed to the recent technological advancements. Interestingly, surgeons are usually less active in the postoperative care of patients with PD because of their surgical duties, ${ }^{1,20,21}$ which is suboptimal for the delivery of medical care and outcome tracking. With systematic tracking, surgeons could offer timely surgical recognition and intervention to optimize postoperative medical care.

In China, the PD population is expected to reach 5 million people by $2030 .{ }^{22}$ Roughly 5000 patients with PD have received DBS surgeries each year, and the number is continuously growing. ${ }^{23}$ In this study, we intended to investigate, from a surgeon's perspective, how remote postoperative assessment could be conducted in patients with PD who had deep brain stimulators implanted; to the best of our knowledge, this has not yet been examined. To provide better postoperative healthcare for patients with PD who received DBS surgery and to allow surgeons improved tracking of surgical outcomes, we used smartphones to perform virtual assessments.

\section{Methods \\ Recruitment}

The Center for Functional Neurosurgery at Ruijin Hospital, Shanghai, has performed more than 1000 PD-related DBS surgeries over the past 20 years. Before surgery, demographic information and motor and nonmotor assessment scores were collected in the database for clinical reference and research purposes. Patients, doctors, and assistants were gathered in a social application. This allowed patients to receive notifications from the doctors and ask questions about specific situations.

For this study, we expected to recruit $\geq 20$ patients with PD for remote assessment. Inclusion criteria included being diagnosed with idiopathic PD and having completed a preoperative motor assessment using the Movement Disorder Society-sponsored revision of the Unified Parkinson's Disease Rating Scale, part III (MDS-UPDRS III). Exclusion criteria included 1) declining to participate, 2) having been assessed in the hospital in the past 6 months, 3) not answering the phone (or the phone number was wrong), 4) not having a $4 \times 1.5-\mathrm{m}$ space at home to perform the motor assessment, 5) having dementia, and 6) death. The local ethics committee approved the study protocol, and all participants provided written informed consent.

Inclusion of patients was either doctor-initiated or patient-initiated. Patients who were within the time window of a postoperative follow-up were considered more urgent for follow-up. Thus, doctor-initiated phone calls were made to invite patients for a virtual assessment. On the other hand, the surgeon sent a notification in the group chat via the social application, stating that the physician assistant would be available for an online assessment. In this way, patients who were not reachable by phone or who had interest in remote assessment could also be included.

\section{Virtual Assessment Tool}

General Information

The virtual assessment was achieved on PD CARE (GYENNO SCIENCE CO., LTD.), an individualized chronic disease management platform. With the PD CARE doctor portal, surgeons and physician assistants are able to provide real-time online consultations and assessments. Before the virtual meeting, patient clinical history records can be rapidly retrieved from the cloud PD clinical information system as a reference. ${ }^{24}$ During virtual visits, surgeons can provide medication and parameter adjustment suggestions. Virtual assessments on motor and nonmotor capabilities can be evaluated by surgeons themselves or physician assistants.

On the patient portal, not only can patients accept postoperative follow-up invitations from the surgeon, but also they can make appointments at their desired time. Furthermore, patients can document their medication intake; record health diaries, including sleep, sports, and meals; and complete self-reported assessment scales to monitor disease progression.

\section{Data Security and Transmission}

The communication mechanism adopts a two-way authentication HTTPS protocol. Sensitive personal information such as passwords and identification cards is encrypted using salting and nonreversible algorithms before being transmitted. Meanwhile, local files and data are protected. Local data are stored in the user's private directory, and lightweight cache data are stored after encryption. Authorization is prompted before the user's local data are obtained.

The remote visit video resolution is 720 pixels (1280 $\times 720$ pixels), and the frame rate is 30 frames/sec. Such high-quality video allows physician assistants to observe each motor task in full detail for accurate scoring.

\section{Study Procedure}

For patients who expressed interest in participating in 
the study, we first made sure that they satisfied the inclusion and exclusion criteria. Then, patients were asked about their disease progression, medication, and occurrence of any complications. If patients and their caregivers were not educated in the use of smartphones, they were invited for nonmotor assessments over the phone, which included the Mini-Mental State Examination (MMSE), Parkinson's Disease Questionnaire 8 (PDQ-8), and 5-level version of the EQ-5D (EQ-5D-5L). Otherwise, patients were invited for both a nonmotor and motor assessment, namely the modified MDS-UPDRS III. These patients were guided through the process of installing PD CARE on their personal device and syncing with the physician assistant to make a 1.5-hour appointment at their convenience in the following 2 weeks. Further instructions for the MDS-UPDRS III, including a video tutorial of each motor task and a document about how MDS-UPDRS III could be conducted at home, were delivered. The physician assistant also instructed the patient to have a pen and a chair with a back and arms and to wear clothes that do not fit too loosely.

On the day of assessment, the physician assistant sent a reminder to patients 15 minutes before the meeting and initiated the video call on PD CARE at the designated time. Patient consent for study participation and video recording was collected at the beginning of the video conference. The physician assistant would first perform the modified MDS-UPDRS III assessment with help from the patient's companion. Afterward, nonmotor assessments were completed orally. Patients were under the medication-on and stimulation-on condition throughout the virtual visit. After the virtual visit, the physician assistant encouraged patients to fill out an anonymous satisfaction questionnaire.

The recorded assessment session was reviewed by two well-trained physician assistants, each rating the scales individually. Results were compared to identify any major discrepancies, and a reevaluation for that specific task was conducted. Afterward, a postassessment report was generated and reviewed by the doctor, which was ultimately delivered to the patient.

\section{Data Collection}

\section{Motor Symptoms}

A modified version of the MDS-UPDRS III was employed to measure motor symptoms of patients with PD. This modified version excluded measurements of rigidity and balance and, thus, had a maximum score of 116. A moderate agreement between clinically and virtually assessed UPDRS III and MDS-UPDRS III was reported, and the modification was proven to be applicable, safe, and qualified for remote assessment by previous studies..$^{14,15,25}$

\section{Nonmotor Symptoms}

Cognitive impairment was measured using the MMSE, and quality of life was measured using both the PDQ-8 and EQ-5D-5L, which was introduced by the EuroQol Group in 2009. These scales are equally applicable in a virtual setting because they include plain questions that result in definitive answers.

\section{Distance, Cost, and Time}

We recorded the patient's home address during the online follow-up visit for cost and time estimations. For patients who lived outside Shanghai, we calculated the cost of a round trip to our hospital by high-speed train and taxi as well as the registration fee to meet with a doctor. For those who lived in Shanghai, we recorded the cost of taking a taxi to commute between home and the hospital. We assumed all patients would return home at the end of the day, so accommodation fees were not included. The amount of time spent was estimated accordingly, with the assumption that there was no wait time. For remote assessment, the internet fee was estimated based on a high standard of charges such that each megabyte of data cost 0.3 Chinese yuan (CNY).

\section{Satisfaction Questionnaire}

We assessed participant opinions on the professionalism of physician assistants, amount of time spent, willingness to participate in long-term virtual follow-ups, and overall satisfaction with the virtual visit using a 4-point Likert scale (very satisfied to unsatisfied). In addition, reasons for participating and the expected frequency of remote visits were collected. There was also space for a free-text response in case the patient had any advice or complaints (Table 1).

\section{Statistical Analysis}

Means and standard deviations were calculated for all parametric data, and percentages were calculated for nonparametric data. Change rates were calculated between pre- and postoperative scale scores and the cost of traditional and virtual visits. A two-tailed paired Student t-test with a $p$ value $<0.05$ reflective of statistical significance was used to assess pre- and postoperative scale score differences and cost differences. Statistical analysis was performed in $\mathrm{R}$ version 4.02 (www.r-project.org).

\section{Results}

Figure 1 provides a descriptive visualization of how the closed-loop virtual visit functions.

\section{Participants}

Patients were invited for a virtual assessment over a 4-week period. Of 332 patients with PD, 49 patients were in the time window of a follow-up and were, thus, contacted by phone. In addition, 24 patients with PD who were not in the exact follow-up time window but who were concerned about disease progression contacted us from the social application. Six patients with PD were excluded for not responding to the phone call, and 4 were excluded because of a wrong telephone number. Two patients had died, and 1 patient was reported by their family as having severe immobility, so they were excluded from the inquiry list.

Of the 60 patients who expressed interest in study participation, 11 withdrew for personal reasons or a strong unwillingness to participate. Four patients reported having technical difficulties such that they were unable to install PD CARE, so we invited them to undergo the nonmotor assessment via telephone. For the remaining 45 patients who reported no technical issues, we delivered further instructions as to how PD CARE was installed, guided them to 
TABLE 1. Satisfaction questionnaire

\begin{tabular}{|c|c|c|c|c|}
\hline & & & Response & \\
\hline 1. Were you satisfied $w /$ today's virtual visit? & Very satisfied & Satisfied & Neither agree nor disagree & Unsatisfied \\
\hline 2. Were you satisfied $w /$ the attitude of the physician assistant? & Very satisfied & Satisfied & Neither agree nor disagree & Unsatisfied \\
\hline 3. Do you think the duration of the virtual visit was reasonable? & Very reasonable & Reasonable & Neither agree nor disagree & Not reasonable \\
\hline \multicolumn{5}{|l|}{ 4. What are the reason(s) that you chose the virtual visit? } \\
\hline 4.1 Inconvenient to reach the hospital & Yes & & No & \\
\hline 4.2 Saving time \& expense & Yes & & No & \\
\hline 4.3 To follow the doctor's arrangement & Yes & & No & \\
\hline 4.4 To try out new technologies & Yes & & No & \\
\hline 5. Are you willing to participate in future virtual visits? & Very interested & Interested & Neither agree nor disagree & Not interested \\
\hline 6. How often should the virtual visit be? & Every mo & Every 3 mos & Every half yr & Every yr \\
\hline \multicolumn{5}{|l|}{$\begin{array}{l}\text { 7. List factors that made you the most satisfied during today's } \\
\text { virtual visit. }\end{array}$} \\
\hline 8. Additional comments & & & & \\
\hline
\end{tabular}

make an appointment with the physician assistant, and sent virtual assessment-related tutorials. After instructions were delivered, 4 patients decided to withdraw, and 22 patients no longer responded to our messages. During the motor assessment, 1 patient was unable to finish the entire process because of fatigue and we were unable to reach her thereafter. Finally, 18 (24.7\%) of the original 73 patients completed both motor and nonmotor assessments via PD CARE, and 4 patients $(5.5 \%)$ completed the nonmotor assessment via phone. Detailed dropout descriptions are provided in Fig. 2 along with a flowchart of the study procedure.

Participants came from 6 provinces throughout China. As shown in Table 2, $11(50 \%)$ of the 22 patients were male and the mean age was 61 years with no significant intergroup age difference. The mean disease duration was 12.28 years, with a mean Hoehn and Yahr (H-Y) stage of 2.78. Participants were well educated, given that the mean duration of education was 13.1 years. Among the 22 patients with PD, 9 (40.91\%) had a college degree or higher. Compared with the general population, our participants were more likely to use the internet at home (100\% vs $86 \%){ }^{14}$

\section{Assessments}

All 22 patients completed the nonmotor assessment. The mean total scores of the MMSE, PDQ- 8 , and EQ5D-5L were $27.64(\mathrm{p}<0.001), 11.58(\mathrm{p}=0.76)$, and $5.76(\mathrm{p}$ $=0.11$ ), respectively. Eighteen patients completed the motor assessment and the mean score of the modified MDSUPDRS III was $25.61(\mathrm{p}=0.02)$.

The duration of the assessment procedure was the length of time from the day we successfully established a connection with the patient to the day the online assessment was completed. On average, it took 3.45 days to complete the process. Moreover, each virtual meeting was recorded and the duration was calculated. The mean duration of the video assessment was 41.3 minutes and that of the nonmotor assessment via phone was 17.5 minutes.

\section{Distance, Cost, and Time}

The mean distance from the patients' homes to the hos- pital was $331.91 \mathrm{~km}$ (Table 3). Two transportation means were used for estimation; high-speed rail and taxi were chosen because of the price stability. The mean estimated cost of an in-person visit, taking the registration fee into consideration, was 427.68 CNY, and it was 20.91 CNY for a virtual visit, assuming that each megabyte of data is worth $0.3 \mathrm{CNY}(\mathrm{p}<0.001)$. However, in real life, some patients may take a flight or intercity bus, which would cause fluctuation of the estimated cost. Additionally, travel time was estimated given the assumption that all patients took high-speed rail or taxi and did not wait before meeting with the doctor. As a result, the mean estimate of time spent was 5.51 hours for an in-person visit and was 0.68 hours for a virtual visit $(\mathrm{p}=0.002)$.

\section{Satisfaction}

A total of 18 patients completed the postassessment satisfaction questionnaire and reported an overall satisfaction (77.78\% very satisfied and $22.22 \%$ satisfied) with the virtual visit (Fig. 3). Patients specifically cited comfort with meeting the physician assistant, the reasonable evaluation time, and a willingness to participate in future virtual visits. All participants acknowledged the great attitude of the physician assistant $(94.44 \%$ very satisfied and $5.56 \%$ satisfied). In addition, in the free-text response section, 15 patients expressed satisfaction and gratefulness that the physician assistant was professional, respectful, and patient. The majority of patients agreed that the evaluation time was reasonable (50\% totally agree, $44.44 \%$ agree, and $5.56 \%$ neither agree nor disagree), and all patients expressed interest in future virtual visits $(61.11 \%$ very willingly and $38.89 \%$ willingly).

For the multiple-choice question regarding the reasons why patients agreed to participate in this novel virtual study, compliance to the surgeon's invitation was the most common reason $(72.22 \%)$. For 10 patients $(55.56 \%)$ inconvenience to the hospital was the driving force, and for 8 patients $(44.44 \%)$ it was a willingness to attempt new technologies, while 5 patients $(27.78 \%)$ reported that the least concerning factors were time and saving money.

Furthermore, 2 patients reported that they experienced 
(2) Remote assessment

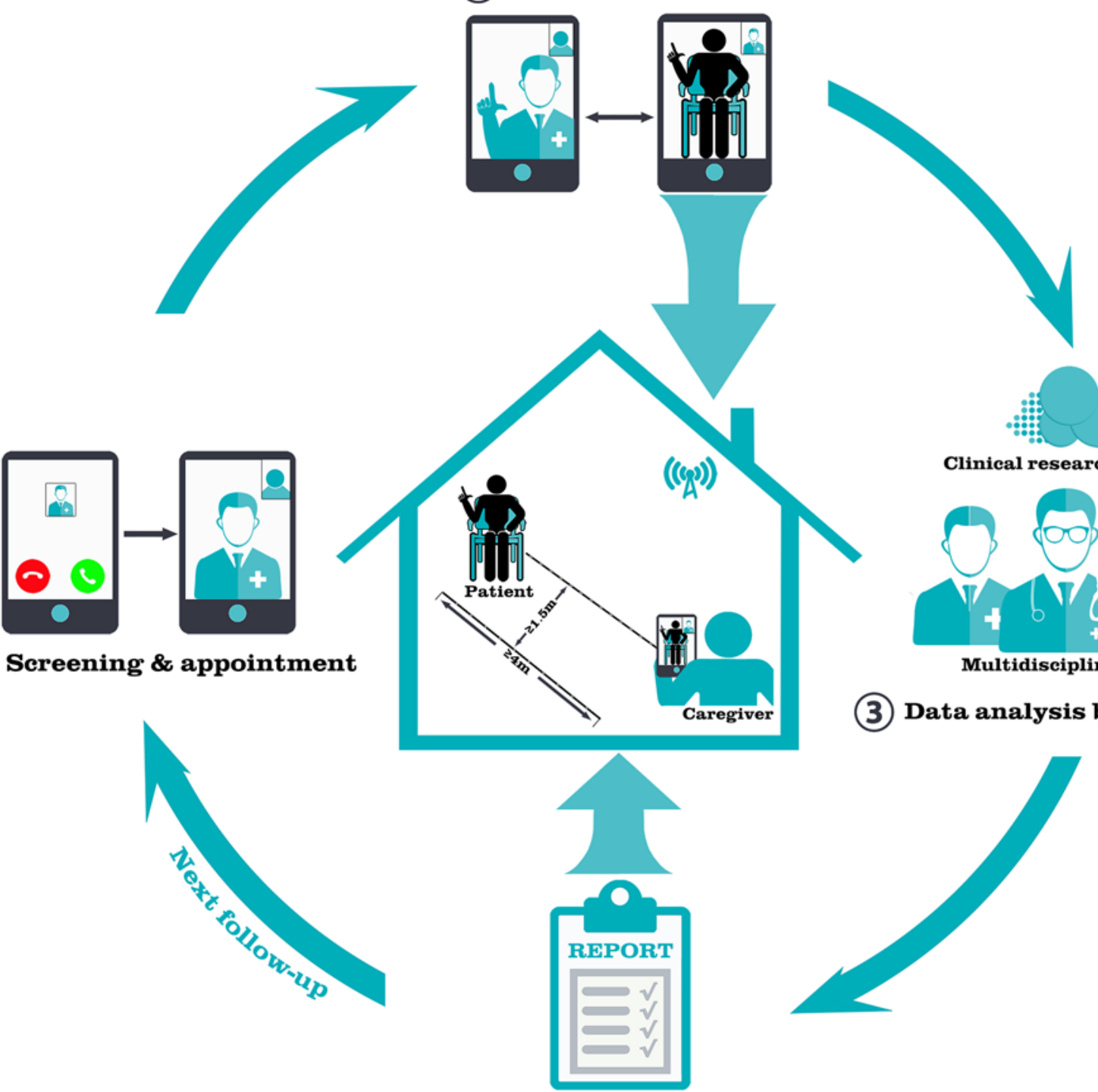

(4) Post-assessment feedback

FIG. 1. Illustration providing an intuitive visualization of how the closed-loop virtual assessment was conducted between patients with PD and the professional team. Patients, with the help of their caregiver, could complete the virtual assessment relatively easily at home. After the assessment, a report summary of the disease progression and advice from a multidisciplinary team, including surgeons, was delivered to the patient, which could positively advance subsequent online follow-up.

a slight network delay, and 1 patient suggested combining teleprogramming with a remote assessment so that the DBS parameters could be accommodated to the patient's recent conditions.

\section{Safety}

No adverse events were observed during the virtual visit.

\section{Discussion}

In this single-center study, 22 patients with PD from different provinces of China were able to complete the vir- tual visit with a physician assistant who specialized in PDrelated assessments. Herein, evidence is provided regarding the advantages of active participation by surgeons, the feasibility of remote assessment for patients with PD who underwent DBS, the substantial time and expense reduction, and a generally positive patient attitude toward innovative follow-up technologies. In addition, we highlighted the applicability of remote assessment of PD DBS by successfully completing the remote patient assessments and receiving positive feedback from patients and caregivers. Furthermore, the estimation of cost reduction provides insights that such an approach is promising for regular, safe, and qualified follow-ups, which has the potential to satisfy 


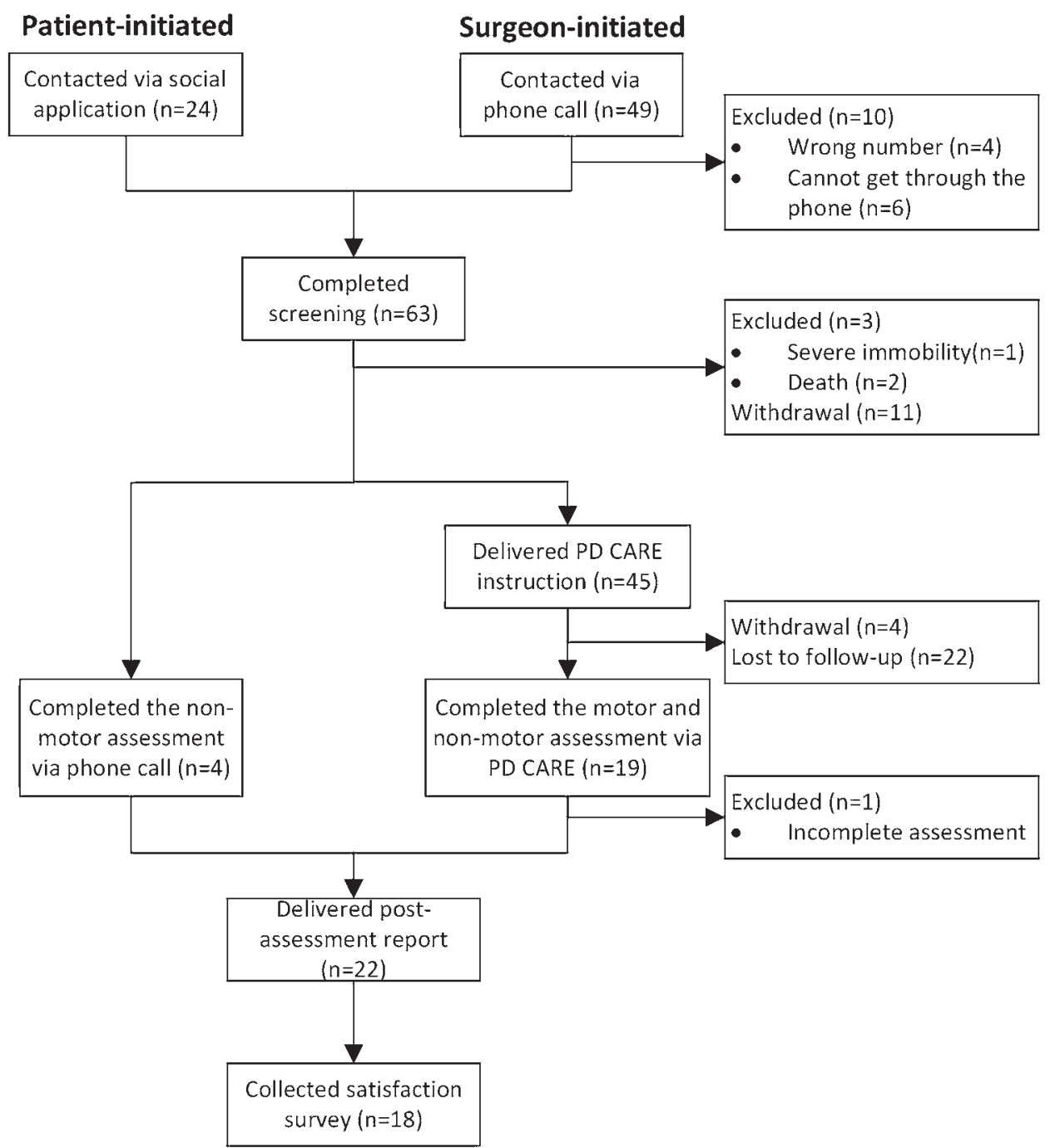

FIG. 2. Flowchart detailing the counts of exclusion, withdrawal, and patients lost to follow-up, as well as the study procedure from the surgeon and physician assistant perspective.

the needs of medical care for patients and research endeavors for healthcare professionals.

Our study agreed with previous research that remote visits could be inspiring and feasible for patients with PD and potentially other neurodegenerative disorders. ${ }^{26-30}$ Our study results suggest that it would be viable and beneficial for surgeons to actively participate in the closed-loop postoperative healthcare of patients with PD via virtual assessment because it allows a closer doctor-patient relationship. Surgeons and physician assistants were able to seamlessly cooperate on the virtual follow-up to create more effective postoperative care. The postassessment report allowed surgeons to revisit for details or as a reference for the next visit. It was significant for surgeons to get first-hand assessment data because they were so experienced that any noticeable phasic changes could be observed, and corresponding advice could be delivered to the patients rapidly.

Beyond the benefits of surgeons being actively involved, the remote assessment was also excellent because of substantial time and travel savings for patients and doctors.
Previous studies have demonstrated the high cost-effectiveness of home-based motor monitoring, suggesting the benefits in clinical information collection and healthcare augmentation for patients with advanced PD. ${ }^{31,32}$ Remote assessment improves access to care in a patient-centered way such that the burden on patients with PD and their caregivers can be minimized. ${ }^{33}$ Similarly, our estimation of the time and expenditure reduction suggests that it is specifically significant for patients who reside in another city or are severely immobile. Meanwhile, the expense reduction is significant for patients who have more financial burdens or need more medical resources to maintain treatment effects. From the surgeon's perspective, the efficient evaluation procedure completed within days could result in huge savings in clinical resources. In addition, as most steps of the remote assessment could be completed by physician assistants, the surgeon's time and attention can be more effectively allocated.

The difference between the preoperative and virtual assessment of the modified MDS-UPDRS III scores was 
TABLE 2. Demographics of patients with PD who underwent DBS

\begin{tabular}{|c|c|}
\hline & Value $(n=22)$ \\
\hline Male sex & $11(50)$ \\
\hline Mean age, yrs & $61(10.78)[36-77]$ \\
\hline Mean education & $13.10(4.05)[6-24]$ \\
\hline \multicolumn{2}{|l|}{ Level of education } \\
\hline Middle school or lower & $6(27.27)$ \\
\hline High school & $6(27.27)$ \\
\hline College or higher & $9(40.91)$ \\
\hline Unclear & $1(4.55)$ \\
\hline \multicolumn{2}{|l|}{ Distance from home to hospital, $\mathrm{km}$} \\
\hline $0-50$ & 7 \\
\hline $50-200$ & 6 \\
\hline $200-500$ & 1 \\
\hline $500-1000$ & 6 \\
\hline $1000-2000$ & 2 \\
\hline Mean disease duration, yrs & $12.28(6.07)[4.81-28.49]$ \\
\hline Mean $\mathrm{H}-\mathrm{Y}$ stage & $2.78(0.90)[1.5-5]$ \\
\hline Mean time after DBS, yrs & $2.29(1.03)[0.76-3.98]$ \\
\hline \multicolumn{2}{|l|}{ DBS targets } \\
\hline Bilat STN & 10 \\
\hline Bilat GPi & 3 \\
\hline Unilat GPi \& STN & 9 \\
\hline \multicolumn{2}{|l|}{ Mean assessment score preop } \\
\hline MMSE & $27.64(2.92)$ [21-30] \\
\hline PDQ-8 & $11.58(4.35)[3-18]$ \\
\hline$E Q-5 D-5 L$ & $5.76(3.65)[0-12]$ \\
\hline Modified MDS-UPDRS III (medication-on) & $25.61(10.20)[10-46]$ \\
\hline Home internet connection & $22(100)$ \\
\hline
\end{tabular}

$\mathrm{GPi}=$ globus pallidus internus; $\mathrm{STN}=$ subthalamic nucleus. Values represent the number of patients (\%) or mean (SD) [range] unless indicated otherwise.
TABLE 3. Estimates of distance, cost of the visit, and amount of time spent

\begin{tabular}{lccr}
\hline & $\begin{array}{c}\text { In-Person } \\
\text { Visit }\end{array}$ & $\begin{array}{c}\text { Virtual } \\
\text { Visit }\end{array}$ & p Value \\
\hline Mean distance from home, $\mathrm{km}$ & 331.91 & 0 & 0.001 \\
\hline Mean visit cost, CNY & 427.68 & 20.91 & $<0.001$ \\
\hline Mean visit time, hrs & 5.51 & 0.68 & 0.002 \\
\hline $\begin{array}{l}\text { A two-tailed paired Student t-test was used to calculate the } p \text { value; } p<0.05 \text { is } \\
\text { statistically significant. }\end{array}$
\end{tabular}

significant. Such decline in motor capabilities is expected, as most previous studies showed an increase of this score (i.e., deterioration in movement) at longer followup periods. ${ }^{34-36}$ In addition, safety, which is always a top concern of patients and caregivers, was well addressed in this study. Several patients reported having experienced falling at least once, but no adverse events were observed during the virtual visit. This is of high importance for patients because they were able to finish the assessment in a much safer environment. And, of course, the virtual visit is significant by reducing the risk of COVID-19 infection, especially for older patients with PD who are considered a high-risk group. ${ }^{37}$

During screening and instruction delivery before the virtual assessment, several patients selected not to participate because of technological challenges. In future studies, relative information about the virtual assessment and application installation could be delivered preoperatively to avoid these problems. For those patients who completed the assessment, the overall high satisfaction provides further proof that virtual assessment could be implemented with confidence. Studies have demonstrated that virtual visits are well received by patients with PD with high satisfaction rates and a particular appreciation for the convenience and comfort afforded. ${ }^{30-32}$ Similarly, the high satisfaction rate in our study suggests the feasibility of im-

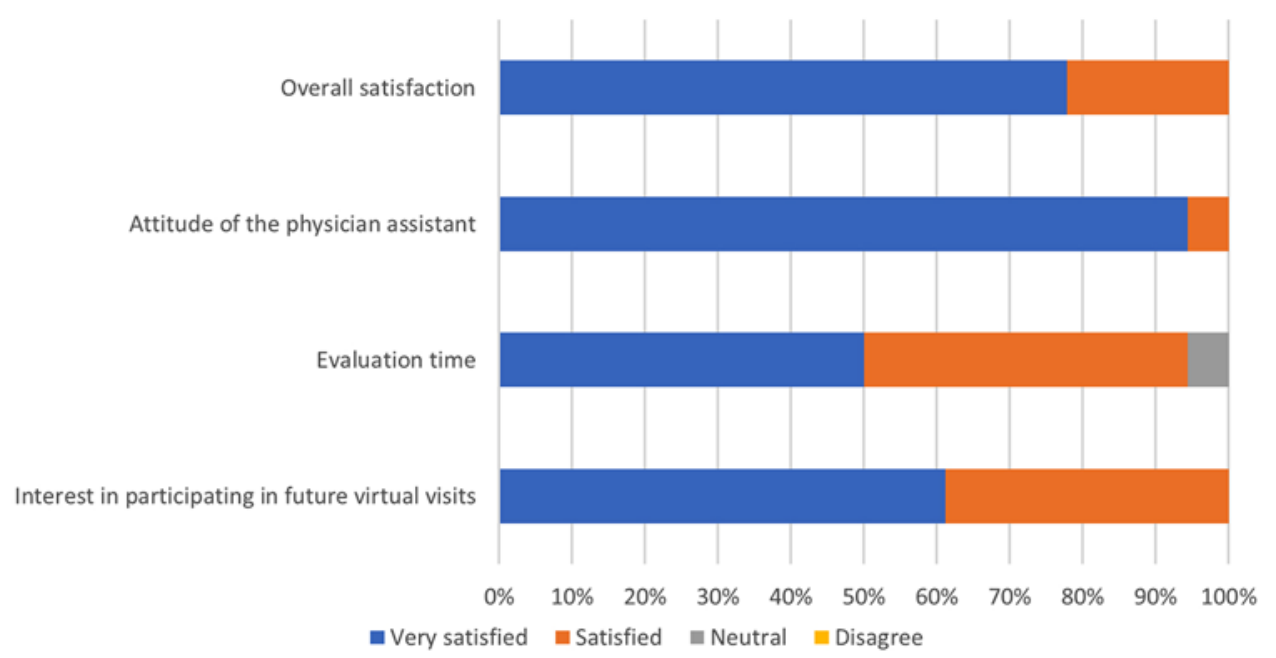

FIG. 3. Bar graph summarizing patient feedback regarding satisfaction with the overall virtual visit, attitude of the physician assistant who conducted the online assessment, and evaluation duration, as well as their willingness to participate in future virtual visits. 
plementing assessment remotely. One potential reason for such high satisfaction is that patients were at home, in their usual environment, which made them feel more relaxed. In addition, the professionalism, patience, and attitude of the physician assistant allowed patients to feel noticed and prioritized. This reflection is desirable because it could be translatable to a smoother realization of long-term virtual care for patients with PD who undergo DBS.

This study has some limitations. First, the assessed population was biased because it was overwhelmingly well educated, given that almost half of the patients had obtained a college degree or higher education level. The potential technological challenges might exclude low-income and rural individuals from completing the virtual motor assessment. Instead of compromising and providing partial assessment via phone calls, it might be more efficient in the long term to educate patients during hospitalization and guide them for installation and the virtual appointment. Second, the remote assessment still required a companion to assist the patient. Lastly, wearable sensors were not applied in this study; in the future, they could be docked into the remote assessment application to assist with motor measurement and even teleprogramming. This is promising as it might assist in formulating a more individualized and well-rounded remote healthcare protocol in the future.

\section{Conclusions}

Innovations in remote assessment technology are highly feasible for their transforming power in the clinical management of PD DBS and research. Video-based remote assessment offers considerable time and resource reduction for both patients and doctors. In addition, it increases safety and is a well accepted and favored tool. Finally, there is the potential to combine such assessment tools with reallife clinical visits and other telemedical technologies to collectively benefit the postoperative healthcare of patients with PD who undergo DBS.

\section{Acknowledgments}

We appreciate all the physician assistants for contacting patients, conducting the remote assessments, and grading the assessment scales. We also want to thank all patients and their caregivers for participating in our study.

\section{References}

1. Bloem BR, Okun MS, Klein C. Parkinson's disease. Lancet. 2021;397(10291):2284-2303.

2. Eberle W, Penders J, Yazicioglu RF. Closing the loop for deep brain stimulation implants enables personalized healthcare for Parkinson's disease patients. Annu Int Conf IEEE Eng Med Biol Soc. 2011;2011:1556-1558.

3. Wielinski CL, Erickson-Davis C, Wichmann R, WaldeDouglas M, Parashos SA. Falls and injuries resulting from falls among patients with Parkinson's disease and other parkinsonian syndromes. Mov Disord. 2005;20(4):410-415.

4. Lyons MK. Deep brain stimulation: current and future clinical applications. Mayo Clin Proc. 2011;86(7):662-672.

5. Chopra A, Klassen BT, Stead M. Current clinical application of deep-brain stimulation for essential tremor. Neuropsychiatr Dis Treat. 2013;9:1859-1865.
6. Wirdefeldt K, Adami HO, Cole P, Trichopoulos D, Mandel J. Epidemiology and etiology of Parkinson's disease: a review of the evidence. Eur J Epidemiol. 2011;26(suppl 1):S1-S58.

7. $\mathrm{Vu} \mathrm{TC}$, Nutt JG, Holford NHG. Progression of motor and nonmotor features of Parkinson's disease and their response to treatment. Br J Clin Pharmacol. 2012;74(2):267-283.

8. Valldeoriola F, Morsi O, Tolosa E, Rumià J, Martí MJ, Martínez-Martín P. Prospective comparative study on costeffectiveness of subthalamic stimulation and best medical treatment in advanced Parkinson's disease. Mov Disord. 2007;22(15):2183-2191.

9. Dams J, Siebert U, Bornschein B, Volkmann J, Deuschl G, Oertel WH, et al. Cost-effectiveness of deep brain stimulation in patients with Parkinson's disease. Mov Disord. 2013; 28(6):763-771.

10. Krack P, Fraix V, Mendes A, Benabid AL, Pollak P. Postoperative management of subthalamic nucleus stimulation for Parkinson's disease. Mov Disord. 2002;17(suppl 3):S188S197.

11. Ross S, Grant A, Counsell C, Gillespie W, Russell I, Prescott $\mathrm{R}$. Barriers to participation in randomised controlled trials: a systematic review. J Clin Epidemiol. 1999;52(12):1143-1156.

12. Ledford H. Coronavirus shuts down trials of drugs for multiple other diseases. Nature. 2020;580(7801):15-16.

13. van den Bergh R, Bloem BR, Meinders MJ, Evers LJW. The state of telemedicine for persons with Parkinson's disease. Curr Opin Neurol. 2021;34(4):589-597.

14. Dorsey ER, Wagner JD, Bull MT, Rizzieri A, Grischkan J, Achey MA, et al. Feasibility of virtual research visits in Fox Trial Finder. J Parkinsons Dis. 2015;5(3):505-515.

15. Tarolli CG, Andrzejewski K, Zimmerman GA, Bull M, Goldenthal S, Auinger P, et al. Feasibility, reliability, and value of remote video-based trial visits in Parkinson's disease. $J$ Parkinsons Dis. 2020;10(4):1779-1786.

16. Arora S, Venkataraman V, Zhan A, Donohue S, Biglan KM, Dorsey ER, Little MA. Detecting and monitoring the symptoms of Parkinson's disease using smartphones: a pilot study. Parkinsonism Relat Disord. 2015;21(6):650-653.

17. Little M, Wicks P, Vaughan T, Pentland A. Quantifying shortterm dynamics of Parkinson's disease using self-reported symptom data from an Internet social network. J Med Internet Res. 2013;15(1):e20.

18. Stillerova T, Liddle J, Gustafsson L, Lamont R, Silburn P. Remotely assessing symptoms of Parkinson's disease using videoconferencing: a feasibility study. Neurol Res Int. 2016; 2016:4802570.

19. Zhang C, Li D, Zeljic K, Tan H, Ning Y, Sun B. A remote and wireless deep brain stimulation programming system. Neuromodulation. 2016;19(4):437-439.

20. Willis AW, Schootman M, Evanoff BA, Perlmutter JS, Racette BA. Neurologist care in Parkinson disease: a utilization, outcomes, and survival study. Neurology. 2011;77(9):851-857.

21. Willis AW, Schootman M, Tran R, Kung N, Evanoff BA, Perlmutter JS, Racette BA. Neurologist-associated reduction in PD-related hospitalizations and health care expenditures. Neurology. 2012;79(17):1774-1780.

22. Lim SY, Tan AH, Ahmad-Annuar A, Klein C, Tan LCS, Rosales RL, et al. Parkinson's disease in the Western Pacific Region. Lancet Neurol. 2019;18(9):865-879.

23. Zhang C, Ramirez-Zamora A, Meng F, Lin Z, Lai Y, Li D, et al. An international survey of deep brain stimulation utilization in Asia and Oceania: the DBS Think Tank East. Front Hum Neurosci. 2020;14:162.

24. Ren K, Liu F, Zhuang H, Ling Y. AI-based multimodal data management and intelligent analysis system for Parkinson's disease: GYENNO PD CIS. In: Proceedings of the 2020 International Symposium on Artificial Intelligence in Medical Sciences. Association for Computing Machinery; 2020: 166-170. 
25. Schneider RB, Myers TL, Tarolli CG, Amodeo K, Adams JL, Jensen-Roberts S, Dorsey ER. Remote administration of the MDS-UPDRS in the time of COVID-19 and beyond. $J$ Parkinsons Dis. 2020;10(4):1379-1382.

26. Bull MT, Darwin K, Venkataraman V, Wagner J, Beck CA, Dorsey ER, Biglan KM. A pilot study of virtual visits in Huntington disease. J Huntingtons Dis. 2014;3(2):189-195.

27. Tarolli CG, Zimmerman GA, Goldenthal S, Feldman B, Berk $\mathrm{S}$, Siddiqi B, et al. Video research visits for atypical parkinsonian syndromes among Fox Trial Finder participants. Neurol Clin Pract. 2020;10(1):7-14.

28. Abdolahi A, Scoglio N, Killoran A, Dorsey ER, Biglan KM. Potential reliability and validity of a modified version of the Unified Parkinson's Disease Rating Scale that could be administered remotely. Parkinsonism Relat Disord. 2013;19(2): 218-221.

29. Dorsey ER, Achey MA, Beck CA, Beran DB, Biglan KM, Boyd CM, et al. National randomized controlled trial of virtual house calls for people with Parkinson's disease: interest and barriers. Telemed J E Health. 2016;22(7):590-598.

30. Beck CA, Beran DB, Biglan KM, Boyd CM, Dorsey ER, Schmidt PN, et al. National randomized controlled trial of virtual house calls for Parkinson disease. Neurology. 2017; 89(11):1152-1161.

31. Korn RE, Wagle Shukla A, Katz M, Keenan HT, Goldenthal $S$, Auinger P, et al. Virtual visits for Parkinson disease: a multicenter noncontrolled cohort. Neurol Clin Pract. 2017; 7(4):283-295.

32. Mammen JR, Elson MJ, Java JJ, Beck CA, Beran DB, Biglan $\mathrm{KM}$, et al. Patient and physician perceptions of virtual visits for Parkinson's disease: a qualitative study. Telemed JE Health. 2018;24(4):255-267.

33. Larson DN, Schneider RB, Simuni T. A new era: the growth of video-based visits for remote management of persons with Parkinson's disease. J Parkinsons Dis. 2021;11(s1):S27-S34.

34. Obeso JA, Olanow CW, Rodriguez-Oroz MC, Krack P, Kumar R, Lang AE. Deep-brain stimulation of the subthalamic nucleus or the pars interna of the globus pallidus in Parkinson's disease. N Engl J Med. 2001;345(13):956-963.

35. Krack P, Batir A, Van Blercom N, Chabardes S, Fraix V, Ardouin C, et al. Five-year follow-up of bilateral stimulation of the subthalamic nucleus in advanced Parkinson's disease. $N$ Engl J Med. 2003;349(20):1925-1934.
36. Weaver FM, Follett KA, Stern M, Luo P, Harris CL, Hur K, et al. Randomized trial of deep brain stimulation for Parkinson disease: thirty-six-month outcomes. Neurology. 2012; 79(1):55-65.

37. Sibley KG, Girges C, Hoque E, Foltynie T. Video-based analyses of Parkinson's disease severity: a brief review. $J$ Parkinsons Dis. 2021;11(s1):S83-S93.

\section{Disclosures}

Dr. Dianyou Li received honoraria and travel expenses from Medtronic, PINS, and SceneRay. Dr. Bomin Sun received research support (donated devices) from SceneRay. Dr. Kang Ren is a stakeholder of GYENNO SCIENCE.

\section{Author Contributions}

Conception and design: Zhang, $\mathrm{Xu}$. Acquisition of data: $\mathrm{Xu}$, Ren. Analysis and interpretation of data: Xu, Zeng. Drafting the article: Xu, Zeng, Qi. Critically revising the article: Xu. Reviewed submitted version of manuscript: Xu. Statistical analysis: Xu, Zeng. Administrative/technical/material support: Zhang, Ren, Sun, Li. Study supervision: Zhang, Ren, Sun, Li.

\section{Supplemental Information \\ Videos}

Video Abstract. https://vimeo.com/622427941.

\section{Online-Only Content}

Supplemental material is available online. Supplemental Table 1. https://thejns.org/doi/suppl/10.3171/ 2021.8.FOCUS21383.

\section{Correspondence}

Chencheng Zhang: Center for Functional Neurosurgery, Ruijin Hospital, Shanghai Jiao Tong University School of Medicine, Shanghai, China.i@cczhang.org. 